\title{
Nachruf
}

\section{Prof. Dr. Hans-Dieter Belitz zum Gedächtnis}

Univ.-Prof. Dr.-Ing. H.-D. Belitz ist nach schwerer Krankheit am 31. März 1993 verstorben.

Geboren am 9. März 1931 in Merseburg studierte er an der Universität Halle (1950-51) und an der Technischen Universität Berlin (1951-1955) Chemie, ging zu Prof. Dr. J. Schormüller an das Institut für Lebensmittelchemie, promovierte 1957 und erhielt 1962 die venia legendi aufgrund einer Habilitationsschrift über Phosphorproteine des Eidotters und ihrem enzymatischen Abbau. Er folgte 1966 einem Ruf an die Technische Universität München auf das Ordinariat für Lebensmittelchemie, wurde zum Vorstand des neu gegründeten Instituts ernannt und wurde 1969 in Personalunion Direktor der Deutschen Forschungsanstalt für Lebensmittelchemie.

In seinem umfangreichen wissenschaftlichen Werk, das in über 200 Veröffentlichungen dargestellt ist, gelang ihm die Aufklärung wichtiger Struktur-Wirkungsbeziehungen bei Proteinen und Geschmacksstoffen.

Die Zusammenhänge zwischen der Struktur und den makroskopischen Eigenschaften von Getreideproteinen verfolgte er seit 1971 in Zusammenarbeit mit dem Kurt-Hess-Institut für Mehl- und Eiweißforschung, das er seit $1973 \mathrm{im}$ Nebenamt geleitet hat. Durch die Sequenzierung charakteristischer Spaltpeptide wurden die Unterschiede bei den wichtigsten Getreidearien sichtbar gemacht und es wurde die Sonderstellung des Weizens hinsichtlich der Backeigenschaften auf molekularer Basis begründet. Gleichzeitig wurden die Strukturen von Getreideproteinen aufgeklärt, die bei genetisch disponierten Personen eine Schleimhautatrophie im Dünndarmbereich (Zöliakie) hervorrufen.

Der Zeitschrift für Ernährungsphysiologie stand Prof. Belitz viele Jahre im wissenschaftlichen Beirat zur Verfügung.

Von der Gesellschaft Deutscher Chemiker wurden seine Leistungen 1988 mit der Verleihung der Joseph-König-Gedenkmünze und von der Arbeitsgemeinschaft Getreideforschung e. V. 1992 mit der Neumann-Medaille gewürdigt.

Sein besonderes Engagement für die Lehre belegt das „Lehrbuch der Lebensmittelchemie", das innerhalb von 10 Jahren viermal aufgelegt und in die englische und spanische Sprache übersetzt worden ist.

Da sein fachkundiger Rat und sein sicheres Urteil sehr geschätzt wurden, war er Mitglied zahlreicher nationaler und internationaler Gremien. Besonders hervorzuheben ist, daß er 21 Jahre den Vorsitz im wissenschaftlichen Ausschuß des Forschungskreises der Ernährungsindustrie inne hatte. In Würdigung seiner ehrenamtlichen Tätigkeiten ist ihm 1985 das Verdienstkreuz am Bande des Verdienstordens der Bundesrepublik Deutschland verliehen worden.

Mit Professor Belitz verliert das Fach Lebensmittelchemie und die Ernährungswissenschaft eine außergewöhnliche Persönlichkeit, deren vorbidliches Engagement für Forschung und Lehre ebenso unvergessen bleiben wird wie sein wissenschaftliches Werk. 